\title{
Prevalencia de síndrome metabólico en pacientes con psoriasis, Centro Dermatológico CES Sabaneta, abril a junio de 2012
}

Metabolic syndrome in patients with psoriasis, Centro Dermatológico CES Sabaneta, April-June 2012

\section{Sol Beatriz Jiménez ${ }^{1}$, Liliana Montoya², Andrés Gaviria³, Daniel Gómez³ ${ }^{3}$ Laura Morales³, Pamela Gallego $^{4}$, Natalia De la Calle ${ }^{4}$}

1. Médica dermatóloga; jefe, Servicio de Dermatología, Universidad CES, Medellín, Colombia.

2. Magíster en Epidemiología; docente, Facultad de Medicina, Universidad CES, Medellín, Colombia.

3. Estudiante de quinto año, Universidad CES, Medellín, Colombia.

4. Médica dermatóloga, Universidad CES, Medellín, Colombia.

\section{RESUMEN}

INTRODUCCIÓN. La psoriasis es una enfermedad inflamatoria, crónica y sistémica, asociada al síndrome metabólico y a factores de riesgo cardiovascular que aumentan la morbilidad de quienes la padecen. Se estima que la prevalencia del síndrome metabólico en la población general varía entre 15 y $20 \%$, y, en el caso de los pacientes con psoriasis, puede aumentar hasta $39 \%$.

OBJETIVO. Establecer la prevalencia del síndrome metabólico en una cohorte de pacientes con diagnóstico o en tratamiento para psoriasis, que consultaron al Centro Dermatológico CES Sabaneta entre el $1^{\circ}$ de abril y el 15 de junio de 2012.

MÉTODOS. Se llevó a cabo un estudio observacional y descriptivo de pacientes con psoriasis que consultaron, al menos una vez, al Centro Dermatológico CES Sabaneta, entre el $1^{\circ}$ de abril y 15 de junio de 2012. Se les hizo el examen físico y se practicaron pruebas de laboratorio para determinar la gravedad de la enfermedad y la presencia de síndrome metabólico, y se calcularon medidas de tendencia central y dispersión, además de proporciones.

RESULTADOS. Se incluyeron 46 pacientes con psoriasis. El $63 \%$ tenía una psoriasis leve y el $37 \%$ tenía formas de moderada a grave; se encontró una prevalencia de síndrome metabólico de 56,5 \%. En aquellos con formas clínicas leves de psoriasis, el síndrome metabólico estuvo presente en 62,1 \% y, en las formas moderadas a graves, en $90 \%$. El 43,5\% de los pacientes con psoriasis y síndrome metabólico tenían un índice normal de masa corporal; el 32,6 \% tenían sobrepeso; el 19,6\% eran obesos y el 4,3\% tenían bajo peso.

CONCLUSIÓN. Los resultados de este estudio demuestran que la psoriasis es una enfermedad sistémica con una alta prevalencia de síndrome metabólico asociado. Este puede estar presente, independientemente de la gravedad de la enfermedad. Aun en las formas leves, se obtuvieron altas prevalencias de síndrome metabólico.

Correspondencia:

Sol Beatriz Jiménez

Email:

sjimenez@ces.edu.co

Recibido: 21 de abril de 2014 .

Aceptado: 20 de octubre de 2014.

No se reportan conflictos de interés.

PALABRAS CLAVE: psoriasis, síndrome metabólico, prevalencia. 


\section{SUMMARY}

BACKGROUND: Psoriasis is a chronic, systemic inflammatory disease, associated with metabolic syndrome and cardiovascular risk, factors that increase the morbidity of patients suffering from it. Prevalence of the metabolic syndrome in the general population varies between 15 and $20 \%$, and in the case of patients with psoriasis it can increase up until $39 \%$.

OBJECTIVE: To determine the prevalence of metabolic syndrome in a cohort of patients diagnosed and/or treated for psoriasis who attended the Centro Dermatológico CES Sabaneta between April $1^{\text {st }}$ and June $15^{\text {th }}, 2012$.

METHODS: A descriptive study of patients with psoriasis who were seen at least once at the Centro Dermatológico CES Sabaneta, between April $1^{\text {st }}$ and June $15^{\text {th }}$, 2012, was carried out. Central tendency measure, dispersion and proportions were calculated.

RESULTS: Forty six patients with psoriasis were included, $63 \%$ of them had a mild psoriasis and 37\% had moderate to severe clinical forms. The prevalence of the metabolic syndrome was 56.5\%: in patients with mild clinical forms of psoriasis was $62.1 \%$, and $90 \%$ in moderate to severe forms. $43.5 \%$ of the patients with psoriasis and metabolic syndrome had a normal corporal mass index, $32.64 \%$ were overweight, and $19.68 \%$ were obese and $4.3 \%$ had low weight

CONCLUSION: This study shows that the study population had a high prevalence of metabolic syndrome associated with psoriasis, but mild forms also have an association with it, not only moderate to severe forms such as described in the literature.

KEY WORDS: psoriasis, metabolic syndrome, prevalence.

\section{INTRODUCCIÓN}

La psoriasis es una enfermedad sistémica inflamatoria, crónica, de la piel, uñas y articulaciones, mediada inmunológicamente ${ }^{1,2}$. Según los datos de la Organización Mundial de la Salud (OMS), afecta entre 1 y $3 \%$ de la población mundial ${ }^{3,4}$. Es una enfermedad de distribución mundial, con una prevalencia global que varía entre 0,6 y $6,5 \%$, siendo mayor en los países nórdicos y menor en los ecuatoriales. En Colombia, el $3 \%$ de las consultas dermatológicas correspondieron a psoriasis ${ }^{5}$. Esta enfermedad se caracteriza por la inflamación crónica y no contagiosa de la piel, que produce placas hiperqueratósicas, escamosas y gruesas, con una amplia variabilidad clínica.

$\mathrm{Su}$ causa etiológica es multifactorial, incluyendo la predisposición genética y los agentes ambientales, así como factores psicológicos, metabólicos, endocrinos, el trauma, el uso de ciertos medicamentos y el padecimiento de enfermedades infecciosas que pueden desencadenar y propagar una respuesta inmunitaria inapropiada ${ }^{6-9}$. Es un trastorno hereditario y se piensa que, probablemente, ocurre por una interacción del sistema inmunitario con las capas profundas de la piel ${ }^{10}$.

La inflamación de la psoriasis es de tipo celular, en la cual hay un estímulo desconocido que activa las células dendríticas presentadoras de antígeno de la piel, que estimulan a las células T ayudadoras de tipo 1 (Th1) que inician una cascada de liberación de citocinas, tales como las interleucinas (IL) 6, 2 y 12, el factor de necrosis tumoral $\propto($ FNT $-\propto)$ y el interferón $\gamma$. Están implicadas también células Th productoras de IL-17 e IL-23. Lo anterior resulta en el reclutamiento y activación de otros tipos de células, como las endoteliales y los neutrófilos, y en la producción de quimiocinas y factores de crecimiento, que llevan a la proliferación y diferenciación anormal de queratinocitos, angiogénesis e inflamación crónica ${ }^{11}$.

En el síndrome metabólico intervienen múltiples factores de riesgo cardiovascular, que incluyen obesidad central, dislipidemia, intolerancia a la glucosa y presión arterial elevada. Se caracteriza por ser un estado proinflamatorio o trombótico, con niveles elevados de IL-6, 
CRITERIOS PARA EL DIAGNÓSTICO DE SÍNDROME METABÓLICO

Obesidad abdominal: circunferencia abdominal mayor de $102 \mathrm{~cm}$ en hombres y de $88 \mathrm{~cm}$ en mujeres.

Elevación de triglicéridos mayor de $150 \mathrm{mg} / \mathrm{dl}$ o estar bajo tratamiento.

Disminución del HDL: menor de $40 \mathrm{mg} / \mathrm{dl}$ en hombres y de $50 \mathrm{mg} / \mathrm{dl}$ en mujeres, o estar bajo tratamiento.

Elevación de la presión arterial: mayor de 130/85 mm de Hg, o estar bajo tratamiento.

Elevación de la glucosa: mayor de $110 \mathrm{mg} / \mathrm{dl}$ o estar bajo tratamiento.

TABLA 1. Criterios para el diagnóstico de síndrome metabólico, según el National Cholesterol Education Program - Adult Treatment Panel III (NCEP ATPIII).

FNT- $\propto$, inhibidor del activador del plasminógeno, fibrinógeno y proteína $\mathrm{C}$ reactiva. La fisiopatología es atribuida a la resistencia a la insulina mediada por adipocitocinas, como el FNT- $\propto$, y disminución de la leptina y la adiponectina ${ }^{12,13,14}$. El exceso de ácidos grasos libres en pacientes con sobrepeso, altera la acción de la insulina en el músculo esquelético y en el hígado, lo que lleva a resistencia a la misma ${ }^{15}$. Esto conduce a la excesiva producción de citocinas proinflamatorias (del tipo Th1) y factores protrombóticos en el tejido adiposo y hepático, incluyendo FNT- $\propto$, IL-6, proteína $C$ reactiva y fibrinógeno. Por lo tanto, este síndrome está asociado a diabetes de tipo 2, enfermedad coronaria, infarto de miocardio y accidente cerebrovascular, y a condiciones inflamatorias como la psoriasis, la artritis reumatoidea y el lupus eritematoso sistémico ${ }^{16}$.

En la TABLA 1 se listan los criterios del síndrome metabólico, según el National Cholesterol Education Program - Adult Treatment Panel III (NCEP ATPIII), los cuales son los más aceptados y aplicables a nuestra población. También existen los criterios de la OMS y los de la Fundación Internacional de la Diabetes, los cuales se basan en parámetros similares.

El diagnóstico se hace con la presencia de tres criterios.

Tanto la psoriasis como el síndrome metabólico comparten la misma falta de regulación inflamatoria, con exceso de producción de citocinas Th1. Se considera que los pacientes con psoriasis están predispuestos a desarrollar síndrome metabólico y, por consiguiente, enfermedad cardiovascular ${ }^{11,17,18,19}$.
También, hay datos de que se encuentra involucrado un factor genético. Los genes PSORS2, PSORS3 y PSORS4 están asociados con propensión a la psoriasis y al síndrome metabólico ${ }^{20}$.

\section{MATERIALES Y MÉTODOS}

Se llevó a cabo un estudio observacional descriptivo de una cohorte de pacientes con psoriasis, que en algún momento consultaron al Centro Dermatológico CES Sabaneta. El objetivo general del estudio fue determinar la prevalencia de síndrome metabólico en los pacientes con diagnóstico o en tratamiento para psoriasis, en el Centro Dermatológico de Sabaneta entre los años 2010 y 2011, además de describir la población según las variables sociodemográficas y clínicas, como hipertensión arterial, diabetes, dislipidemia, las distintas formas clínicas de psoriasis y tiempo de evolución de la enfermedad, y los hábitos de vida que pudieran desencadenar el síndrome metabólico, como el consumo de cigarrillo y licor, y el ejercicio físico.

Dicho estudio se llevó a cabo entre el $1^{\circ}$ de abril y el 15 de junio de 2012. Para la muestra se incluyeron aquellos pacientes que estaban en las bases de datos del servicio, que al momento constaba de 285 . Solo se lograron contactar y aceptaron participar en el estudio 53 pacientes, que fue la población estudiada. De estos 53, sólo se incluyeron 46 , ya que en los 7 restantes no se practicaron las pruebas de laboratorio.

Se consideraron los siguientes criterios de inclusión: diagnóstico previo de psoriasis de cualquier tipo y gravedad, disponibilidad del paciente para responder la encuesta, evaluación clínica y práctica de los exámenes de laboratorio. Se excluyeron los que no aceptaron participar.

Los pacientes fueron citados para evaluación por dos dermatólogos y se les hizo una encuesta. Se sometieron a un examen físico completo y se les ordenaron exámenes de laboratorio. Se diseñó un instrumento en Excel (Office Excel ${ }^{\circledR}$ compatible con Windows 7) que permitió recolectar de forma adecuada todas las variables establecidas

\section{(TABLA 2).}

Para la medición de la gravedad de la psoriasis, se tomó en cuenta el índice PASI (Psoriasis Area and Severity Index), así: formas leves, puntuaciones entre o y 10; y moderadas a graves, mayores de $10^{5,12}$. El PASI discrimina la gravedad en leve, menor de 10 , y moderada a grave, mayor de 10; no hace subclasificaciones, ni clasifica la psoriasis en moderada a partir de valores de 5 .

La encuesta de recolección de datos incluyó todas las variables demográficas y clínicas. En los datos del 

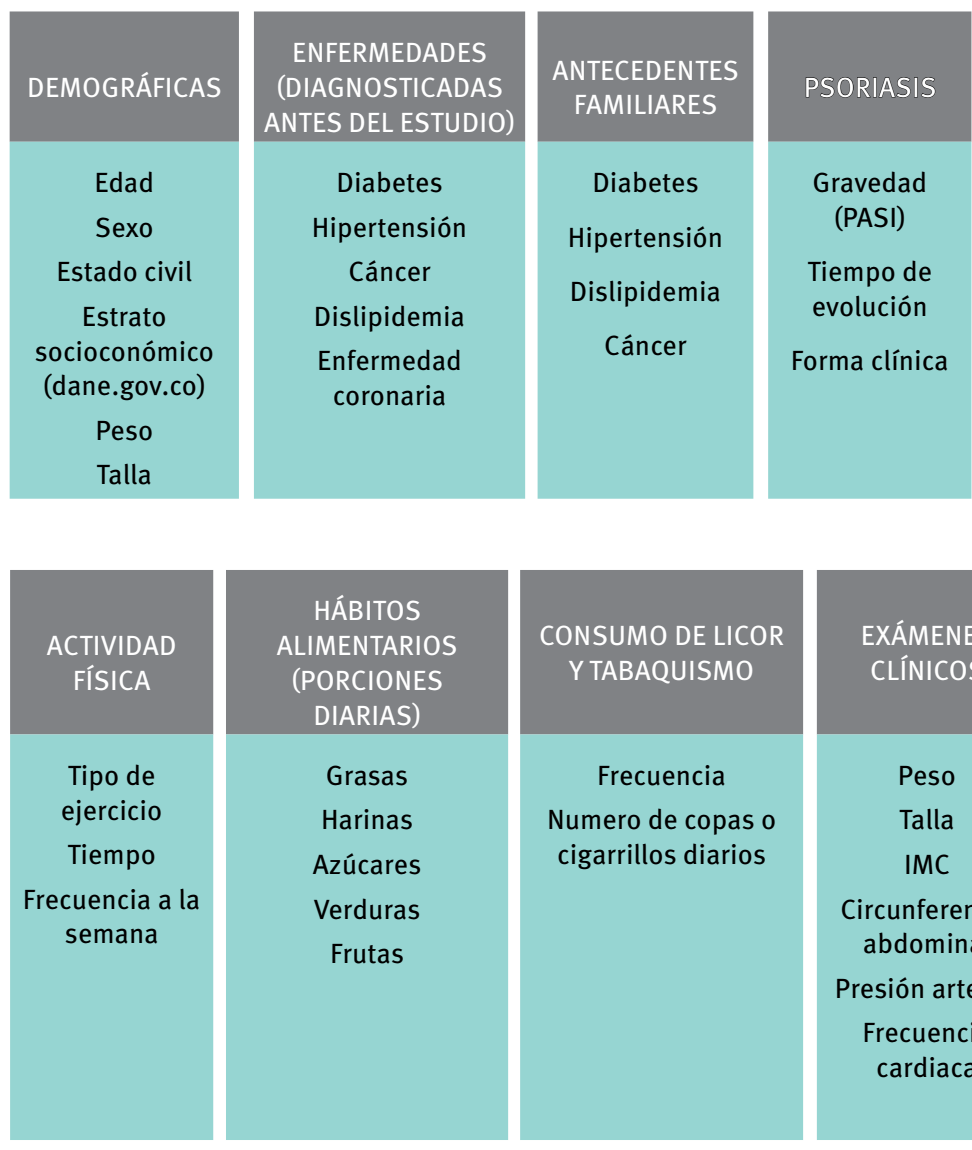

TABLA 2. Variables del estudio.

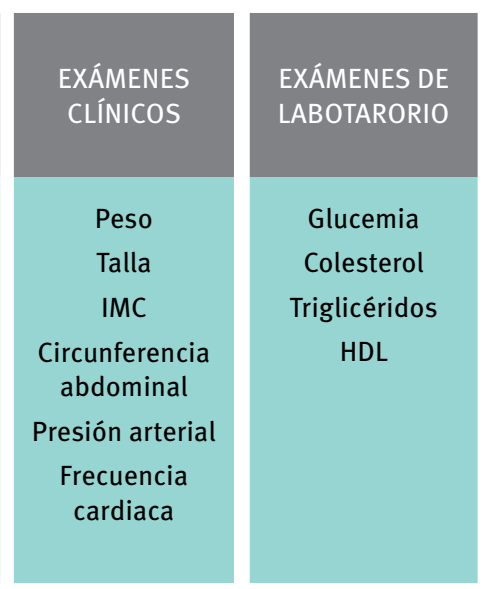

examen físico se incluyó el índice de masa corporal (IMC); para su medición, se dividió el peso en kg por la talla en $\mathrm{m}^{2} \mathrm{y}$ se interpretó así: 18,5 o menos, peso bajo; de 18,5 a 24,99, peso normal; de 25 a 29,99, sobrepeso; y mayor de 30, obesidad. Se investigaron las formas de alimentación de cada uno de los pacientes, discriminando la ingestión diaria en número de carnes, grasas, harinas, verduras, frutas y dulces. Entre las variables de laboratorio, se incluyeron las mediciones de colesterol total, triglicéridos, HDL, LDL y glucemia.

El diagnóstico de síndrome metabólico se hizo con base en los criterios del NCEP ATPIII, porque eran los que más se adecuaban a nuestra población ${ }^{21,22}$. El proyecto fue enviado a la Universidad CES, el cual fue aprobado por el Comité Operativo de Investigaciones y de Ética de la Facultad de Medicina, y el estudio fue conducido según los principios éticos de la declaración de Helsinki. El análisis estadístico se hizo con el programa SPSS ${ }^{\circledR}$, versión 8.

Para las variables cuantitativas se calcularon las medidas de tendencia central, como la media y la mediana, y de dispersión, como la varianza y la desviación estándar.

Para las variables cualitativas, se tuvieron en cuenta medidas como las proporciones o las razones.

Se calculó la prevalencia de síndrome metabólico en aquellos pacientes con diagnóstico o tratamiento para psoriasis, con su respectivo intervalo de confianza.

\section{RESULTADOS}

De los 46 pacientes incluidos en el estudio, 28 (63\%) eran hombres y 17 (37\%), mujeres, en edades comprendidas entre los 14 y los 73 años, con promedio de 50,71 años. El $60,9 \%$ vivía en estrato 3. El 10,9\% de los pacientes tenía antecedentes personales de diabetes, el $37 \%$, de hipertensión arterial, el 43,5\%, de dislipidemia, y el 6,5\%, de enfermedad coronaria. Ninguno tenía antecedentes de 
cáncer. El 45,7 \% de la población tenía historia familiar de enfermedad coronaria.

Las formas clínicas de psoriasis estaban distribuidas así: en placas, $82,6 \%$, en gotas, 2,2\%, y palmo-plantar, $2,2 \%$. El tiempo promedio de evolución de la enfermedad fue de 14,2 años (rango, o,30-53). El $63 \%$ de los pacientes tenía una psoriasis leve y, el $37 \%$, formas moderadas a graves.

De la población estudiada, 14 (30,4 \%) pacientes consumían alcohol, de los cuales la mitad prefería el aguardiente, con un promedio de 8,3 copas, 1,3 veces por semana. El 28,6 \% de los pacientes con un PASI moderado a grave consumía licor, pero no se observó una diferencia estadísticamente significativa entre el PASI de los que consumían licor y el de los que no lo hacían. Respecto al consumo de cigarrillo, 35 (76,1\%) pacientes no eran fumadores y de los 11 (23,9\%) restantes, 3 iniciaron su consumo antes de los 18 años y, en promedio, consumían 5,2 cigarrillos al día (rango, 2-12 cigarrillos al día). El 27,3 $\%$ de los fumadores presentaba PASI moderado a grave, en comparación con el $25,7 \%$ de los no fumadores, y no se observó una diferencia estadísticamente significativa.

Los resultados obtenidos sobre los hábitos de vida saludable mostraron que $15(32,6 \%)$ pacientes no hacian ejercicio, $28(60,9 \%)$ caminaban, $2(4,3 \%)$ trotaban y $1(2,2 \%)$ practicaba el ciclismo. El promedio de veces por semana era: en los que caminaban, 4,89 veces, 44,82 minutos; en los que trotaban, 5 veces a la semana, 45 minutos en promedio, y en el que practicaba ciclismo, 5 veces por semana, 90 minutos.

La media del índice de masa corporal fue de $26 \mathrm{~kg} / \mathrm{m}^{2}$, con una desviación estándar de 5,o (rango, 16,41 a 38,37).
El 43,5\% de la población en estudio tenía un IMC normal; el 32,6 \% sufría sobrepeso, el 19,6 \% eran obesos y el 4,3\% tenía bajo peso.

Los valores obtenidos en los parámetros para determinar el síndrome metabólico, se presentan en la FigURA 1. Se obtuvo una prevalencia general de síndrome metabólico en la población en estudio de $56,5 \%$ ( $58,8 \%$ en mujeres y 55,2 \% en hombres) y, cuando se cruzó con el PASI (gravedad), se obtuvo 62,1 \% para las formas leves y $90 \%$ para las formas moderadas a graves. De los pacientes con psoriasis sin síndrome metabólico, $60 \%$ tenía un IMC normal, $25 \%$, sobrepeso, 10\%, peso bajo, y $5 \%$, obesidad. De aquellos con psoriasis y síndrome metabólico, el 30,8 \% tenía un IMC normal, el $38,4 \%$, sobrepeso, y el 30,8 \%, obesidad.

\section{DISCUSIÓN}

La psoriasis es una enfermedad sistémica asociada a otras concomitantes como el síndrome metabólico, con muchos efectos deletéreos en la calidad de vida de las personas y una menor tasa de supervivencia a 10 años. El sufrir de psoriasis aumenta de manera independiente $6,2 \%$ el riesgo absoluto de sufrir en los siguientes 10 años un evento cardiovascular mayor ${ }^{23}$. El tratamiento para la psoriasis contribuye a la reducción de varios factores de riesgo cardiovascular tales como la inflamación y el estrés oxidativo, lo cual puede disminuir las probabilidades de dichos eventos ${ }^{14}$.

Se estima que la prevalencia del síndrome metabólico en la población general varía de 15 al 20 \% y, en los casos

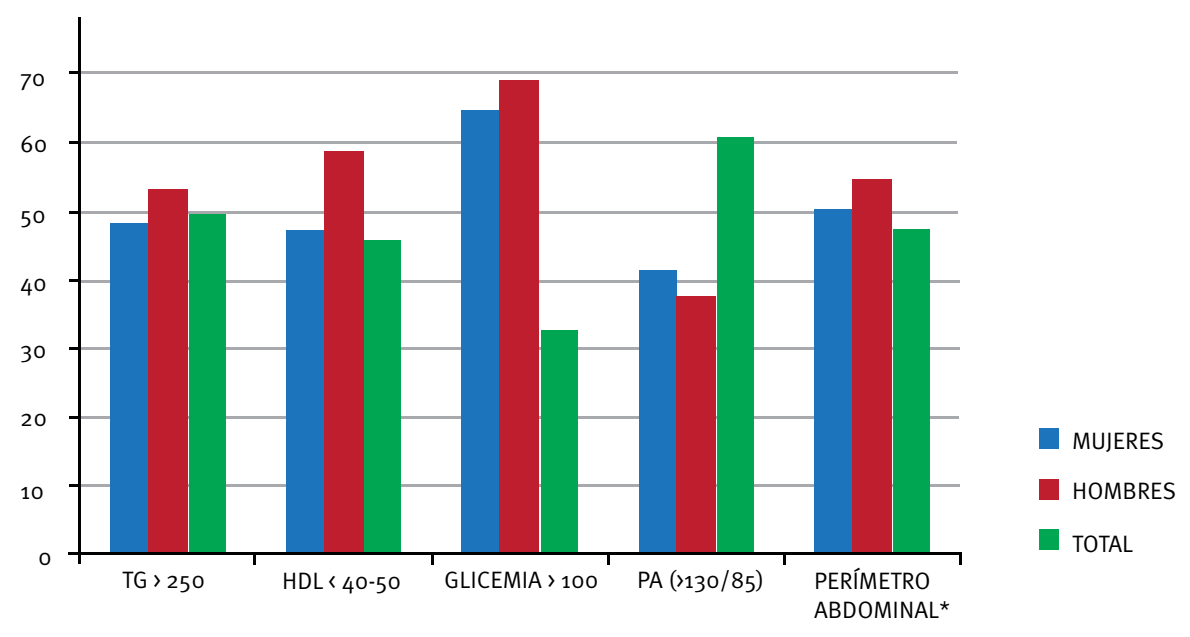

\section{FiguRA 1.}

Criterios para el síndrome metabólico discriminados según sexo.

TG: triglicéridos; HDL: high density lipoprotein; PA: presión arterial

^ $\mathrm{H}:>90 \mathrm{~cm}, \mathrm{M}:>80 \mathrm{~cm}$ 
de psoriasis, puede aumentar hasta $39 \% \%^{24}$. En América Latina se estima que una de cada tres o cuatro personas mayores de 20 años presenta criterios diagnósticos para síndrome metabólico; la prevalencia aumenta con la edad y es más frecuente en las mujeres ${ }^{25}$.

De los cinco criterios diagnósticos de síndrome metabólico, la obesidad abdominal y la hiperlipemia o dislipidemia (aumento de los triglicéridos y disminución de la HDL), son los factores más importantes en casos de asociación de este síndrome con psoriasis. En general, se ha estimado que la asociación con algunos de los criterios oscila entre 1,7 y 2,0 veces. En Colombia, los reportes de Rojas, et al., en una población de pacientes con psoriasis y síndrome metabólico en la ciudad de Bogotá, reportó una prevalencia de 59,6\%, muy semejante a la obtenida en nuestra población (56,5\%). En otros estudios, como el de Gisondi, et al., se obtuvo una prevalencia de 30,1 \%, en comparación con una de 20,6 \% en la población de control ( $\mathrm{p}=0,005 ; \mathrm{OR}=1,65$, intervalo de confianza de $95 \%: 1,16-2,35)$, comparada con la población colombiana ${ }^{14,26}$.

En este estudio, el síndrome metabólico fue más frecuente en hombres, a diferencia de la población de Bogotá, en la cual fue más prevalente en mujeres ${ }^{27,28}$.

La edad promedio fue de 50 años, lo que podría estar relacionado más frecuentemente con el síndrome metabólico, asociado, además, a malos hábitos alimentarios, ejercicio, tabaquismo y consumo de licor, y psoriasis ${ }^{12,6,16}$. De los pacientes sin psoriasis, el $30 \%$ de los mayores de 50 años y hasta el $40 \%$ de los mayores de 6o, pueden presentar síndrome metabólico ${ }^{28}$.

La mayoría de los pacientes de este estudio presentaba una psoriasis leve, como en el estudio de Rojas, et al., y en las estadísticas mundiales ${ }^{5}$, pero aun así, mostraban una mayor prevalencia de síndrome metabólico, asociada a mayor riesgo cardiovascular. Esta asociación es controvertida, ya que Mehta, et al., y Malbris, et al., reportaron que la psoriasis por sí misma conlleva un incremento en la mortalidad por enfermedad cardiovascular ${ }^{1,2,6,29,30}$. La relación exacta entre la inflamación cutánea y el síndrome metabólico no está completamente entendida, aunque se ha observado que los cambios metabólicos no son una consecuencia exclusiva de la enfermedad cutánea de larga evolución, sino que, de hecho, pueden preceder el inicio de la psoriasis ${ }^{31}$. Por otro lado, Gisondi, et al., Mebazaa, et al., y Nisa, et al., no encontraron correlación entre la gravedad de psoriasis y la prevalencia de síndrome metabólico ${ }^{12,13,20}$. A pesar de que la mayoría de nuestros pacientes tenía formas leves de psoriasis, presentaban una alta prevalencia de síndrome metabólico, estableciendo que este podría estar presente en los pacientes con psoriasis, independientemente de la gravedad de la enfermedad.

El 52\% de nuestra población tenía un IMC elevado, de los cuales $32 \%$ estaban en sobrepeso y $19,6 \%$ eran obesos, a pesar de que la mayoría realizaban actividad física de manera regular, con un promedio de cinco veces por semana. Hay información sustancial que indica que la psoriasis se relaciona con un riesgo incrementado de obesidad; sin embargo, se desconoce la relación de causalidad. En algunos estudios de cohorte se sugiere que la obesidad es un factor de riesgo para el desarrollo de psoriasis. No obstante, los resultados de grandes bases de datos sobre psoriasis señalan el aumento de peso como posterior al inicio de la enfermedad.

Henseler y Christophers reportaron una correlación positiva entre psoriasis y obesidad, igual que en un gran número de estudios transversales, donde observaron que un IMC alto coincide con formas más graves de psoriasis $^{20,31,32}$. Naldi, et al., en un estudio de 2.00o pacientes, encontraron que los obesos (criterio para síndrome metabólico) tenían una psoriasis más grave y eran más resistentes al tratamiento, comparados con los no obesos ${ }^{32}$. Hossler, et al., "observaron dos pacientes con un índice de masa corporal mayor de $50 \mathrm{~kg} / \mathrm{m}^{2}$ que mejoraron significativamente de la psoriasis después de cirugía bariátrica y pérdida de peso" 33 .

No obstante, Herron, et al., encontraron que los pacientes con psoriasis eran dos veces más propensos a ser obesos, comparados con la población general ${ }^{34}$. Más tarde, Naldi, et al., identificaron la obesidad como un factor de riesgo independiente asociado a la psoriasis, habiendo $16 \%$ de obesos al momento del diagnóstico de psoriasis ${ }^{32}$.

En un importante estudio prospectivo de cohorte en mujeres jóvenes, se confirmó que la obesidad precedía a la psoriasis, encontrándose que el peso corporal estaba directamente asociado con el riesgo de desarrollar psoriasis $^{35,36}$. Se sabe que la obesidad está asociada con un grado de inflamación persistente caracterizado por incrementos en los niveles de leptina, resistina, IL-6, TNF- $\propto$, IL-8 y MCP-1, los cuales podrían aumentar el grado de inflamación en la psoriasis ${ }^{35,37}$. Además, la psoriasis se acompaña de hiperhomocisteinemia, factor de riesgo que propicia la disfunción endotelial, causando aterosclerosis y trombosis venosa ${ }^{38,39}$.

Esta población realizaba una actividad física adecuada y frecuente, tenía un consumo de licor y cigarrillos relativamente bajo $\mathrm{y}$, a pesar de esto, la prevalencia de síndrome metabólico fue alta, por lo que la psoriasis podría considerarse como un factor desencadenante de enfermedad metabólica ${ }^{1,2,6,16}$. La mayoría de la población era de estrato 3 (según la clasificación 
en Colombia, corresponde al medio-bajo: usuarios con menores recursos y beneficiarios de subsidios en los servicios públicos domiciliarios).

Existen reportes donde se establece la relación entre la condición socioeconómica y los hábitos alimentarios, como el de Regidor, et al., en población adulta española; comparando las variables del síndrome metabólico con los factores socioeconómicos, se encontró una mayor frecuencia de este síndrome, especialmente en mujeres que presentaban condiciones socioeconómicas más bajas y mayor grado de sedentarismo ${ }^{40}$.

Se podría decir que a mejor nivel social, mayor consumo de proteínas y menor de harinas. En Medellín, se sabe que la base del aporte calórico son los cereales y derivados, y existe una relación directamente proporcional entre el estrato socioeconómico y el consumo de carbohidratos, e inversamente proporcional con las proteínas ${ }^{41}$. La población estudiada demostró mayor ingestión de harinas, baja cantidad de proteínas, dulces, huevos y grasas insaturadas, lo que lleva a un desequilibrio nutricional, que en nuestra población se reflejó en sobrepeso y obesidad, factores predisponentes para el desarrollo de síndrome metabólico.

En un estudio de Takahashi, et al., sobre el síndrome metabólico y la dieta, se concluyó que el consumo de grasas saturadas y fibra disminuye el riesgo cardiovascular $^{9,42}$. La alteración concomitante más frecuente en nuestros pacientes fue la dislipidemia, como lo reportado en estudios previos, en los cuales se ha demostrado su asociación ${ }^{33,35}$, además de preceder en algunos casos el inicio de la psoriasis ${ }^{43}$. Aunque no se ha confirmado una relación directa entre psoriasis y dislipidemia, Akhyani y Gottlieb demostraron que en la psoriasis es más común encontrar niveles altos de lípidos, hecho que puede ser responsable de la alta prevalencia de accidentes cardiovasculares en estos pacientes. Esto puede explicarse por el efecto que tienen los adipocitos sobre las citocinas proinflamatorias, como el FTN- $\propto$ y la IL-6, las cuales aumentan y alteran el nivel de ácido+s grasos libres, colesterol y lípidos ${ }^{23}$.

Muchos de nuestros pacientes (10,9\%) eran diabéticos y posiblemente los valores de glucemia se encontraban en rangos normales, ya que estaban en tratamiento. La relación entre psoriasis y resistencia a la insulina o diabetes mellitus de tipo 2, se ha demostrado especialmente en mujeres, relacionada con un IMC elevado, como lo reflejado en este estudio ${ }^{16,17,23,34,44}$. Hay mayor prevalencia de resistencia a la insulina en pacientes con psoriasis. Kaye, et al., encontraron que la psoriasis podía aumentar el riesgo del desarrollo de diabetes ${ }^{4,35}$. La asociación entre psoriasis y diabetes de tipo 2, es aún mayor. En múltiples estudios transversales se encontró que la pso- riasis, especialmente la más grave, otorgaba un riesgo mayor (2,48 veces más) de presentar diabetes ${ }^{35,45}$. En un estudio de casos y controles en Israel, se encontró que los pacientes con psoriasis tuvieron $27,9 \%$ de prevalencia de diabetes mellitus, mientras que en el grupo de control fue de $19,5 \%{ }^{43}$. También, se observó una mayor predisposición a hipertensión arterial ( 4 \% en psoriáticos y 37,2 \% en controles) ${ }^{43}$. El 29,4\% de los pacientes con psoriasis eran obesos, en comparación con $23,5 \%$ de los controles, y hubo dislipidemia en 50,9 \% de los enfermos, en comparación con 44,2 \% de los controles ${ }^{43}$.

En diversos estudios se ha demostrado una asociación entre psoriasis y dislipidemia aterogénica, con aumento en las concentraciones de triglicéridos en plasma, VLDL y LDL, y disminución de $\mathrm{HDL}^{30,33}$. Esto involucra una mayor incidencia de infartos miocárdicos, tromboflebitis, embolia pulmonar y accidentes cerebrovasculares. Desde el punto de vista metabólico, la psoriasis puede ser un factor de riesgo agresivo, aun en pacientes jóvenes. Esto hace indispensable el diagnóstico temprano y el manejo oportuno, para evitar complicaciones y enfermedades concomitantes, y mejorar la calidad de vida de los pacientes.

La pérdida de peso por medio de restricción calórica, mejora las enfermedades metabólicas concomitantes, y disminuye la resistencia a la insulina, los lípidos en suero y las cifras de presión arterial ${ }^{35}$.

Algunos medicamentos utilizados en la psoriasis, como los retinoides o la ciclosporina, pueden inducir dislipidemia o empeoramiento del perfil metabólico del paciente $^{17}$. Otros, como las estatinas, no sólo ayudan a modular algunos elementos del síndrome metabólico como la dislipidemia, sino que han demostrado disminuir la respuesta inflamatoria, mejorando, en algunos casos, las manifestaciones de la psoriasis ${ }^{14}$.

\section{CONCLUSIÓN}

La psoriasis no puede considerarse una condición de la piel solamente, sino más bien, una enfermedad inflamatoria sistémica, que se asocia con varias enfermedades cardio-metabólicas concomitantes y aumento del riesgo cardiovascular. El médico debe tener presente esta combinación, para estar atento a detectar los síntomas tempranos y hacer un seguimiento clínico y de laboratorio en todos los casos de psoriasis, independientemente de su gravedad y, en especial, en aquellos con enfermedades de larga evolución. El dermatólogo debe trabajar en equipo con otros especialistas, para poder proporcionarles a sus pacientes un tratamiento óptimo que produzca un mayor impacto en su enfermedad y mejore el pronóstico. 


\section{REFERENCIAS}

1. Davidovici BB, Sattar N, Prinz JC, Jörg PC, Puig L, Emery P, et al. Psoriasis and systemic inflammatory diseases: Potential mechanistic links between skin disease and co-morbid conditions. J Invest Dermatol. 2010;130:1785-96.

2. Lin HW, Wang KH, Lin HC, Lin HC. Increased risk of acute myocardial infarction in patients with psoriasis: A 5-year population-based study in Taiwan. J Am Acad Dermatol. 2011;64:495-501.

3. Love TJ, Qureshi AA, Karlson EW, Gelfand JM, Choi HK. Prevalence of the metabolic syndrome in psoriasis: Results from the National Health and Nutrition Examination Survey, 2003-2006. Arch Dermatol. 2011;147:419-24.

4. Kaye JA, Li L, Jick SS. Incidence of risk factors for myocardial infarction and other vascular diseases in patients with psoriasis. Br J Dermatol. 2008;159:895-902.

5. González C, Londoño A, Castro L. Guías basadas en la evidencia para el manejo de la psoriasis en Colombia. Bogotá: Panamericana Formas e Impresos; 2012.

6. Zindanci I, Albayrak O, Kavala M, Kocaturk E, Can B, Sudogan $\mathrm{S}$, et al. Prevalence of metabolic syndrome in patients with psoriasis. Scientific World Journal. 2012;2012:312463.

7. Shelling ML, Federman DG, Prodanovich S, Kirsner RS. Psoriasis and vascular disease: An unsolved mystery. Am J Med. 2008;121:360-5.

8. El-Mongy S, Fathy H, Abdelaziz A, Omran E, George S, Neseem $\mathrm{N}$, et al. Subclinical atherosclerosis in patients with chronic psoriasis: A potential association. J Eur Acad Dermatol Venereol. 2010;24:661-6.

9. Takahashi H, lizuka H. Psoriasis and metabolic syndrome. J Dermatol. 2012;39:212-8.

10. Vélez N, Bohórquez L, Corrales MF, Orrego S, Jiménez SB, Zuluaga A, Ochoa FL. Caracterización de los pacientes con psoriasis en tratamiento con fototerapia. Rev Asoc Colomb Dermatol. 2011;19:100-6.

11. Ryo M, Nakamura T, Kihara S, Kumada M, Shibazaki S, Takahashi M, et al. Adiponectin as a biomarker of the metabolic syndrome. Circ J Off J Jpn Circ Soc. 2004;68:975-81.

12. Fu LW, Vender R. Systemic role for vitamin D in the treatment of psoriasis and metabolic syndrome. Dermatol Res Pract. 2011;2011:276079.

13. Hulthe J, Hultén LM, Fagerberg B. Low adipocyte-derived plasma protein adiponectin concentrations are associated with the metabolic syndrome and small dense low-density lipoprotein particles: Atherosclerosis and insulin resistance study. Metabolism. 2003;52:1612-4.

14. Ghazizadeh R, Tosa M, Ghazizadeh M. Clinical improvement in psoriasis with treatment of associated hyperlipidemia. Am J Med Sci. 2011;341:394-8.

15. Eckel RH, Grundy SM, Zimmet PZ. The metabolic syndrome. Lancet. 2005;9468:1415-28.

16. Alsufyani MA, Golant AK, Lebwohl M. Psoriasis and the metabolic syndrome. Dermatol Ther. 2010;23:137-43.

17. Dreiher J, Weitzman D, Davidovici B, Shapiro J, Cohen AD. Psoriasis and dyslipidaemia: A population-based study. Acta Derm Venereol. 2008;88:561-5.
18. Augustin M, Reich K, Glaeske G, Schaefer I, Radtke M. Comorbidity and age-related prevalence of psoriasis: Analysis of health insurance data in Germany. Acta Derm Venereol. 2010;90:147-51.

19. Ghazizadeh R, Shimizu H, Tosa M, Ghazizadeh M. Pathogenic mechanisms shared between psoriasis and cardiovascular disease. Int J Med Sci. 2010;7:284-9.

20. Azfar RS, Gelfand JM. Psoriasis and metabolic disease: Epidemiology and pathophysiology. Curr Opin Rheumatol. 2008;20:416-22.

21. Kimball AB, Guérin A, Tsaneva M, Yu AP, Wu EQ, Gupta SR, et al. Economic burden of comorbidities in patients with psoriasis is substantial. J Eur Acad Dermatol Venereol. 2011;25:157-63.

22. Barona MI, Escobar CE. Psoriasis y parapsoriasis En: Falabella R, Victoria J, Barona MI, Domínguez L. Fundamentos de Medicina Dermatológica. Séptima edición. Medellín: Corporación para Investigaciones Biológicas; 2009. p. 64-9.

23. Mehta NN, Yu Y, Pinnelas R, Krishnamoorthy P, Shin DB, Troxel $\mathrm{AB}$, et al. Attributable risk estimate of severe psoriasis on major cardiovascular events. Am J Med. 2011;124:775.e1-6.

24. Londoño A, González C, Castro L, Puig Ll. Psoriasis y su relación con el síndrome metabólico. Rev Colomb Reumatol. 2013;20:228-36.

25. Rosas J, González A, Aschner P, Bastarrachea B. Epidemiología, diagnóstico, control, prevención y tratamiento del síndrome metabólico en adultos. Consensos ALAD. 2010;18:25-44.

26. Gisondi P, Tessari G, Conti A, Piaserico S, Schianchi S, Peserico $\mathrm{A}$, et al. Prevalence of metabolic syndrome in patients with psoriasis: A hospital-based case-control study. Br J Dermatol. 2007;157:68-73.

27. Rojas R, Castellanos H, Peñaranda E. Prevalencia del síndrome metabólico, gravedad clínica y calidad de vida en pacientes con psoriasis del Hospital Universitario de La Samaritana. Rev Asoc Colomb Dermatol. 2013;21:220-5.

28. Reyes MA, Paternina H, Garzón H. Guías síndrome metabólico. Primera edición. Bogotá: Thomson PLM, S.A.; 2009. p. 234-47.

29. Gelfand JM, Neimann AL, Shin DB, Wang X, Margolis DJ, Troxel AB. Risk of myocardial infarction in patients with psoriasis. JAMA. 2006;296:1735-41.

30. Wakkee M, Thio HB, Prens EP, Sijbrands EJG, Neumann HAM. Unfavorable cardiovascular risk profiles in untreated and treated psoriasis patients. Atherosclerosis. 2007;190:1-9.

31. Martyn-Simmons CL, Ranawaka RR, Chowienczyk P, Crook MA, Marber MS, Smith CH, et al. A prospective case-controlled cohort study of endothelial function in patients with moderate to severe psoriasis. Br J Dermatol. 2011;164:26-32.

32. Naldi L, Addis A, Chimenti S, Giannetti A, Picardo M, Tomino $\mathrm{C}$, et al. Impact of body mass index and obesity on clinical response to systemic treatment for psoriasis. Evidence from the Psocare project. Dermatol Basel Switz. 2008;217:365-73.

33. Hossler EW, Maroon MS, Mowad CM. Gastric bypass surgery improves psoriasis. J Am Acad Dermatol. 2011;65:198-200.

34. Herron MD, Hinckley M, Hoffman MS, Papenfuss J, Hansen CB, Callis KP, et al. Impact of obesity and smoking on psoriasis presentation and management. Arch Dermatol. 2005;141:1527-34.

35. Gisondi P, Ferrazzi A, Girolomoni G. Metabolic comorbidities and psoriasis. Acta Dermatovenerol Croat ADC. 2010;18:297-304.

36. Setty AR, Curhan G, Choi HK. Obesity, waist circumference, weight change, and the risk of psoriasis in women: Nurses' Health Study II. Arch Intern Med. 2007;167:1670-5. 
37. Johnston A, Arnadottir S, Gudjonsson JE, Aphale A, Sigmarsdottir AA, Gunnarsson SI, et al. Obesity in psoriasis: Leptin and resistin as mediators of cutaneous inflammation. Br J Dermatol. 2008;159:342-50.

38. Cakmak SK, Gül U, Kiliç C, Gönül M, Soylu S, Kiliç A. Homocysteine, vitamin B12 and folic acid levels in psoriasis patients. J Eur Acad Dermatol Venereol. 2009;23:300-3.

39. Christophers E. Comorbidities in psoriasis. Clin Dermatol. 2007;25:529-34.

40. Regidor E, Gutiérrez-Fisac JL, Banegas JR, Domínguez V, Rodríguez-Artalejo F. Influencia a lo largo de la vida de las circunstancias socioeconómicas, de la inactividad física y de la obesidad sobre la presencia de síndrome metabólico. Rev Esp Salud Pública. 2007;81:25-31.
41. McDonald I, Connolly M, Tobin AM. A review of psoriasis, a known risk factor for cardiovascular disease and its im pact on folate and homocysteine metabolism. J Nutr Metab. 2012;2012:965385.

42. Mallbris L, Akre O, Granath F, Yin L, Lindelöf B, Ekbom A, et al. Increased risk for cardiovascular mortality in psoriasis inpatients but not in outpatients. Eur J Epidemiol. 2004;19:225-30.

43. Singh G, Aneja SPS. Cardiovascular comorbiditiy in psoriasis. Indian J Dermatol. 2011;56:553-6.

44. Gottlieb AB, Chao C, Dann F. Psoriasis comorbidities. J Dermatol Treat. 2008;19:5-21.

45. Cohen AD, Dreiher J, Shapiro Y, Vidavsky L, Vardy DA, Davidovici B, et al. Psoriasis and diabetes: A population-based crosssectional study. J Eur Acad Dermatol Venereol. 2008;22:585-9. 